\title{
Long non-coding RNA ZNF674-AS1 regulates miR-23a/E-cadherin axis to suppress the migration and invasion of non-small cell lung cancer cells
}

\author{
Jie Wang ${ }^{1, \#}$, Sha Liu ${ }^{3 \#}$, Tao Pan ${ }^{3 \#}$, Mingkun Wang ${ }^{3}$, Liang $\mathrm{Li}^{2}$, Xiangqian Weng ${ }^{3}$, Panhong Jia ${ }^{4}, \mathrm{Qi} \mathrm{Li}^{2}$, \\ Xiangdong Zhou ${ }^{1,2}$
}

${ }^{1}$ Nanjing Medical University, Nanjing, China; ${ }^{2}$ Department of Respiratory Diseases, the First Affiliated Hospital of Hainan Medical University, Haikou, China; ${ }^{3}$ Department of Radiotherapy, the First Affiliated Hospital of Hainan Medical University, Haikou, China; ${ }^{4}$ Hainan Medical University, Haikou, China

Contributions: (I) Conception and design: J Wang, S Liu, Q Li, X Zhou; (II) Administrative support: M Wang; (III) Provision of study materials or patients: J Wang, T Pan; (IV) Collection and assembly of data: All authors; (V) Data analysis and interpretation: J Wang, X Zhou; (VI) Manuscript writing: All authors; (VII) Final approval of manuscript: All authors.

"These authors contributed equally to this work.

Correspondence to: Qi Li; Xiangdong Zhou. Department of Respiratory Diseases, the First Affiliated Hospital of Hainan Medical University, 31 Longhua Road, Longhua District, Haikou 570100, China. Email: lqlq198210@sina.com; longman185@163.com.

Background: Non-small cell lung cancer (NSCLC) is one of the most common malignant tumors. The prognosis of patients with advanced NSCLC is poor due to metastasis. In recent years, the role of long noncoding RNAs (lncRNAs), a class of non-coding RNA molecules, in NSCLC has become an increasingly popular focus of studies. This study aimed to investigate the effects of ZNF674-AS1 and microRNA (miR)23a on the migration and invasion abilities of NSCLC cells in vitro and explore the underpinning molecular mechanisms.

Methods: The expression levels of ZNF674-AS1 and miR-23a in NSCLC tissues and cells were detected by quantitative real-time polymerase chain reaction (qRT-PCR). Scratch test and transwell test were used to detect the effects of ZNF674-AS1 and miR-23a on the migration and invasion of NSCLC cells. The luciferase reporter gene experiment was used to verify miRNA targets. Western blot experiments were used to analyze protein expression.

Results: ZNF674-AS1 was downregulated in NSCLC tissues and cells, and inhibited the migration and invasion of NSCLC cells in vitro. In contrast, the expression of miR-23a, a downstream target of ZNF674AS1, was increased in NSCLC tissues and cells. We found that miR-23a could antagonize the role of ZNF674-AS1 in NSCLC. E-cadherin was identified as a downstream target gene of miR-23a, and miR-23a could directly inhibit its expression.

Conclusions: ZNF674-AS1 inhibits the migration and invasion of NSCLC cells by regulating a miR-23a/ E-cadherin axis. ZNF674-AS1 and miR-23a could become potential therapeutic targets for NSCLC.

Keywords: Non-small cell lung cancer (NSCLC); long non-coding RNA (lncRNA); ZNF674-AS1; miR-23a; E-cadherin

Submitted Jul 16, 2021. Accepted for publication Aug 30, 2021.

doi: $10.21037 /$ tcr-21-1499

View this article at: https://dx.doi.org/10.21037/tcr-21-1499 


\section{Introduction}

Non-small cell lung cancer (NSCLC) is one of the most common malignant tumors and claims more lives than any other cancer in the world $(1,2)$. At present, surgery, chemotherapy, and radiotherapy constitute the main treatment options for patients with NSCLC. However, the efficacy of these traditional treatment strategies for patients with advanced disease is extremely limited $(3,4)$. Advanced NSCLC mainly owes its poor prognosis to metastasis. Therefore, clarifying the molecular mechanism of tumor metastasis is critical in the development of new treatment strategies (5).

Long non-coding RNAs (lncRNAs) are recently discovered non-coding RNAs with a length of more than $200 \mathrm{nt}$, which are potential regulators of numerous cellular processes (6). Through various molecular mechanisms, lncRNAs perform a range of biological functions, including the binding of microRNAs (miRNAs), the regulation of RNA binding proteins (RBPs), and the promotion of messenger RNA (mRNA) transcription (7). An increasing number of studies have shown that lncRNAs are abnormally expressed in various tumors and play an essential regulatory role in tumor cell proliferation, differentiation, invasion, and metastasis (8). For instance, a study by Yao et al. reported that the IncRNA JHDM1D-AS1 was upregulated in NSCLC and was significantly correlated with tumor stage and lymph node metastasis (9). Further, it was found to act as an independent prognostic factor for the overall survival of patients with NSCLC. At the mechanistic level, the authors observed that JHDM1D-AS1 promoted the growth and metastasis of NSCLC by binding and stabilizing the DHX15 protein in tumor cells (9). Wang et al. observed that another IncRNA, LINC01116, was upregulated in gefitinib-resistant NSCLC cells and tissues and played a key role in the resistance of NSCLC cells to gefitinib by affecting the expression of IFI44 (10).

A recent study showed that 1ncRNA ZNF674-AS1 could induce G0/G1 cell cycle arrest of NSCLC cells by antagonizing miR-423-3p and promoting p21 expression, thus inhibiting tumor growth (11). However, the effect of ZNF674-AS1 on NSCLC metastasis is unknown. Our study predicts that miR-23a is a new target of ZNF674AS1. Studies have shown that the expression level of miR$23 \mathrm{a}$ in non-small cell lung cancer tissues is significantly up-regulated, which is an important prognostic factor for NSCLC patients (12). Therefore, using previous studies as a basis, we set out to further explore the role of ZNF674-
AS1 and miR-23a in NSCLC metastasis and the related regulatory pathways, with the aim of improving the understanding of the mechanism of NSCLC metastasis in order to find potential molecular therapeutic targets.

We present the following article in accordance with the MDAR reporting checklist (available at https://dx.doi. org/10.21037/tcr-21-1499).

\section{Methods}

\section{Tissue specimens}

Forty pairs of NSCLC tumor tissues and corresponding adjacent normal tissues were used in this study. All the tissues were collected from patients who underwent surgical resection of NSCLC in the First Affiliated Hospital of Hainan Medical University and were confirmed by postoperative pathology. None of the patients had received any anticancer treatment before receiving surgery. Written informed consent was obtained from each patient. Approval for the collection and application of patient specimens was granted by the ethics committee of the First Affiliated Hospital of Hainan Medical University (No.: 2019-57). All procedures performed in this study involving human participants were in accordance with the Declaration of Helsinki (as revised in 2013).

\section{Cell culture and transfection}

The NSCLC cell lines A549 and PC9 and the human normal lung epithelial cells BEAS-2B were supplied by American Type Culture Collection (ATCC; Manassas, VA, USA). All cell lines were cultured in Roswell Park Memorial Institute (RPMI) 1640 medium (Thermo Fisher Scientific) supplemented with $10 \%$ fetal bovine serum (FBS) at $37{ }^{\circ} \mathrm{C}$ in a cell incubator containing $5 \%$ carbon dioxide. Cell transfection was performed using Lipofectamine 3000 transfection reagent (Invitrogen).

\section{Quantitative real-time polymerase chain reaction ( $q R T$ - PCR)}

TRIzol reagent (Invitrogen, CA, USA) was used to isolate total RNA from the tissues and cell lines. RNA was reversetranscribed into complementary DNA (cDNA) using the High-Capacity cDNA Reverse Transcription Kit (ThermoFisher Scientific). The ABI 7900 system (Applied Biosystems, CA, USA) and SYBR pre-mix Ex Taq (Takara) 
were used for quantitative real-time PCR (qRT-PCR). With GAPDH and U6 as an internal reference, the $2^{-\triangle \Delta C T}$ method was applied to calculate the relative expression of ZNF674AS1 and miR-23a. The primer sequence used was ZNF674AS1, forward, 5'-CCATGCCAGCATTTGCTATTCA-3', reverse, 5'-GGGCTTGAGGCTCTAAAGATGT-3'; GAPDH, forward, 5'-GGGAAACTGTGGCGTGAT-3', reverse, 5'-GAGTGGGTGTCGCTGTTGA-3'. miR-23a, forward, 5'-GGGGTTCCTGGGGATGGGATTT-3', reverse, 5'-TGGTGTCGTGGAGTCG-3'; U6, forward, 5'-CTCGCTTCGGCAGCACATATACT-3'; reverse, 5'-ACGCTTCACGAATTTGCGTGTC-3'.

\section{Wound-healing assay}

The migration ability of NSCLC cells was examined by scratch test. Cells were seeded on a 6-well plate and cultured to $90 \%$ fusion. Then, a scratch was made in the cell monolayer with the tip of a sterile pipette. The cells were washed three times with phosphate-buffered saline (PBS) to remove cell debris and then cultured in serum-free RPMI 1640 Medium without serum for 24 hours. At 0 and 24 hours, a light microscope was used to photograph the scratch. The scratch closure rate was taken to represent the relative migration ability of the cells.

\section{Transwell assay}

Transwell experiments were conducted to evaluate the invasion ability of NSCLC cells. The experiments were performed using $8-\mu \mathrm{m}$ Transwell chambers (Corning, MA, USA), which were pre-coated with Matrigel (BD Biosciences, NJ, USA). NSCLC cells were suspended in RPMI 1640 Medium without FBS and inoculated into the upper chamber of the Transwell. The lower chamber was filled with a complete medium containing $10 \%$ FBS. After 24 hours' culture, the cells that had passed through the membrane were fixed with methanol, stained with crystal violet, and photographed and counted under a microscope.

\section{Luciferase reporter assay}

The pmirGLO vector was used to construct pmirGLOZNF674-AS1-wt (wild type), pmirGLO- ZNF674AS1-mut (Mutant), pmirGLO-E-Cadherin 3'UTR (three prime untranslated region)-wt, and pmirGLO-Ecadherin 3'UTR-mut fluorescence reporter gene plasmids. Subsequently, the luciferase plasmids and miR-23a mimics were co-transfected into NSCLC cells using Lipofectamine 3000 transfection reagent. After 48 hours' culture, a dualluciferase reporter analysis system (Promega, WI, USA) was used to measure the luciferase activity of the cells.

\section{Western blot analysis}

Cellular proteins were extracted with RIPA lysis buffer (Sigma-Aldrich ). Protein samples were separated by sodium dodecyl sulfate-polyacrylamide gel electrophoresis and transferred to a polyvinylidene difluoride membrane. The membrane was sealed with $5 \%$ skim milk for 1 hour at room temperature and incubated with anti-E-cadherin antibody (ab76055, Abcam, USA) overnight at $4{ }^{\circ} \mathrm{C}$. Subsequently, the membrane was incubated with secondary antibodies (Cell Signaling Technology, MA, USA) for 2 hours at room temperature. An enhanced chemiluminescence kit (ThermoFisher Scientific) was used to display the protein bands.

\section{Statistical analysis}

Data were analyzed using SPSS18.0 software (IBM, IL, USA) and GraphPad Prism 5 (GraphPad Software, CA, USA). Each experiment was repeated at least three times independently, and the data were expressed as average \pm standard deviation. Differences between two groups were analyzed by Student's $t$-test, and single-factor variance analysis was used for comparisons between two groups. $\mathrm{P}<0.05$ was recognized as a statistically significant difference.

\section{Results}

ZNF674-AS1 is downregulated in NSCLC tissues and cell lines

First, we examined the expression levels of ZNF674-AS1 in NSCLC tissues and cell lines. The results showed that ZNF674-AS1 was expressed at a significantly lower in NSCLC tissues than in adjacent normal tissues (Figure 1A). The expression of ZNF674-AS1 in the A549 and PC9 NSCLC cell lines was also considerably lower than that in the standard lung epithelial cell line BEAS-2B (Figure 1B). These results indicate that the expression of ZNF674-AS1 is downregulated in NSCLC. To further investigate its biological role in NSCLC cells, ZNF674AS1 was overexpressed and silenced in A549 and PC9 cells 

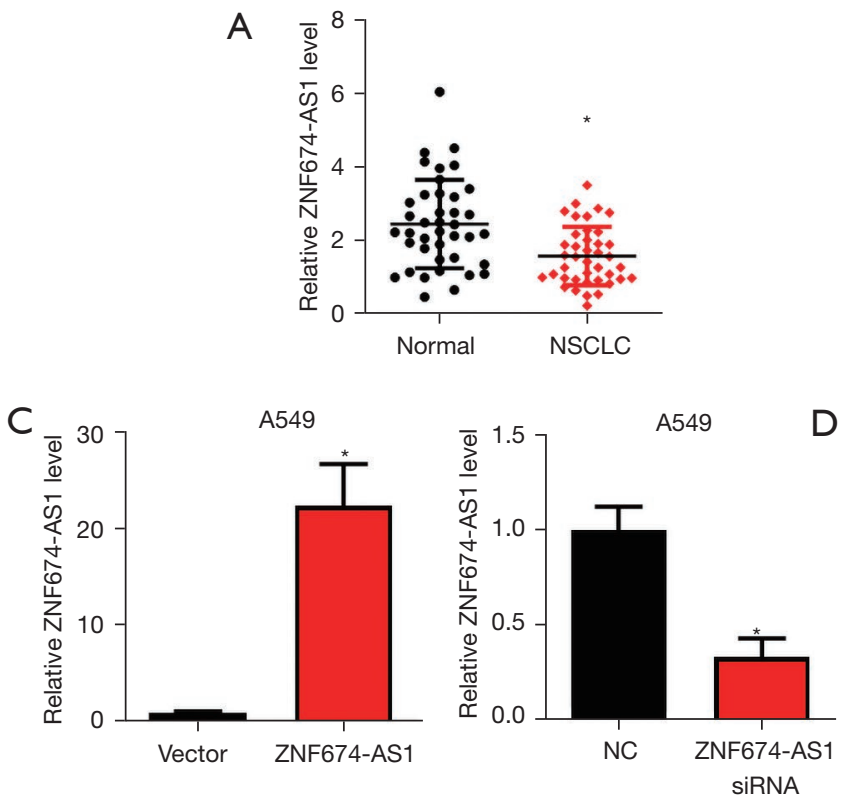
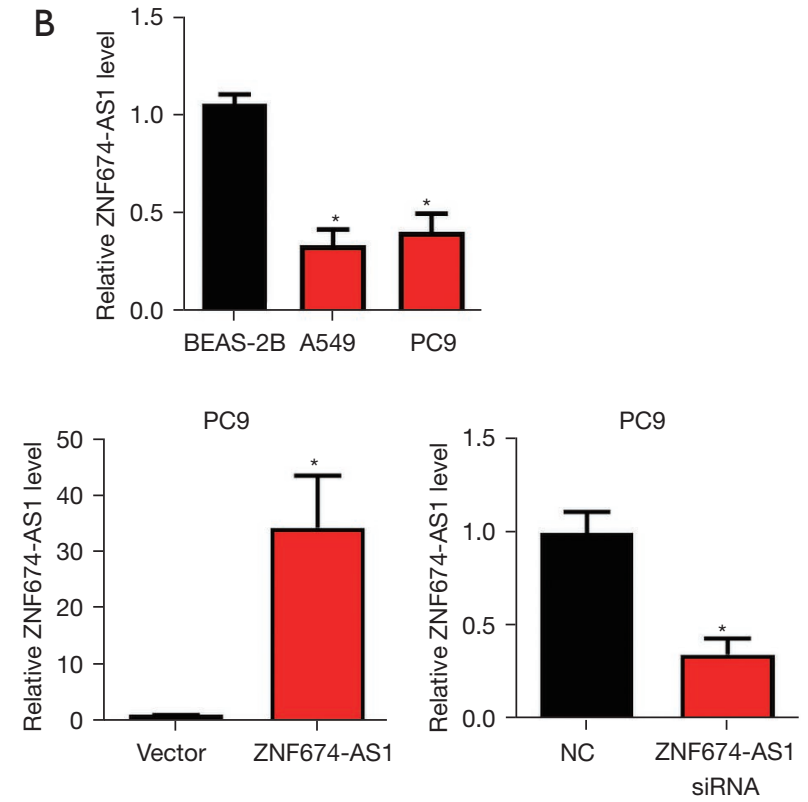

Figure 1 The expression of ZNF674-AS1 is downregulated in NSCLC tissues and cell lines. (A) The expression of ZNF674-AS1 was detected by qRT-PCR. (B) The expression of ZNF674-AS1 in the NSCLC cell lines A549 and PC9 and the normal lung epithelial cell line BEAS-2B was detected by qRT-PCR. (C,D) The overexpression and silencing of ZNF674-AS1 in A549 and pC9 cells. *, P<0.05. NSCLC, non-small cell lung cancer; qRT-PCR, quantitative real-time polymerase chain reaction.

(Figure 1C,1D).

\section{ZNF674-AS1 inbibits the migration and invasion abilities of NSCLC cells}

Subsequently, we investigated the effects of ZNF674-AS1 on NSCLC cell migration and invasion by performing scratch and Transwell assays, respectively. The scratch test results showed that overexpressing ZNF674-AS1 in A549 and pC9 cells significantly inhibited NSCLC cell migration (Figure 2A), while silencing ZNF674-AS1 promoted cell migration (Figure 2B). Similarly, the Transwell assay results showed that overexpression of ZNF674-AS1 significantly inhibited the invasion ability of A549 and PC9 cells (Figure 2C), while silencing ZNF674-AS1 had the opposite effect (Figure 2D). These results suggested that ZNF674AS1 can inhibit the migration and invasion of NSCLC cells.

\section{miR-23a is a downstream target of ZNF674-AS1}

Through searching the Starbase database, we predicted miR-23a to be one of the direct downstream targets of ZNF674-AS1 (Figure 3A). The predicted binding sequence between miR-23a and ZNF674-AS1 was confirmed by double luciferase reporter gene experiment (Figure 3B). Our results indicated that miR-23a is indeed a downstream target of ZNF674-AS1. Subsequently, we detected the expression levels of miR-23a in NSCLC tissues and cells, with qRT-PCR revealing them to be significantly downregulated (Figure 3C,3D).

miR-23a antagonizes the role of ZNF674-AS1 in NSCLC

Next, we transfected miR-23a mimics into NSCLC cells overexpressing ZNF674-AS1. Scratch and Transwell experiment results showed that the inhibitory effect of ZNF674-AS1 overexpression on NSCLC cell migration and invasion could be eliminated by miR-23a mimics (Figure $4 A, 4 B$ ). Additionally, we transfected miR-23a inhibitor into ZNF674-AS1-silenced NSCLC cells. The results of scratch and Transwell experiments showed that the enhancement of NSCLC cell migration and invasion caused by ZNF674-AS1 silencing could be eliminated by miR-23a inhibitor (Figure $4 C, 4 D$ ). The above results suggest that ZNF674-AS1 exerted its inhibitory effect by targeting miR-23a. 

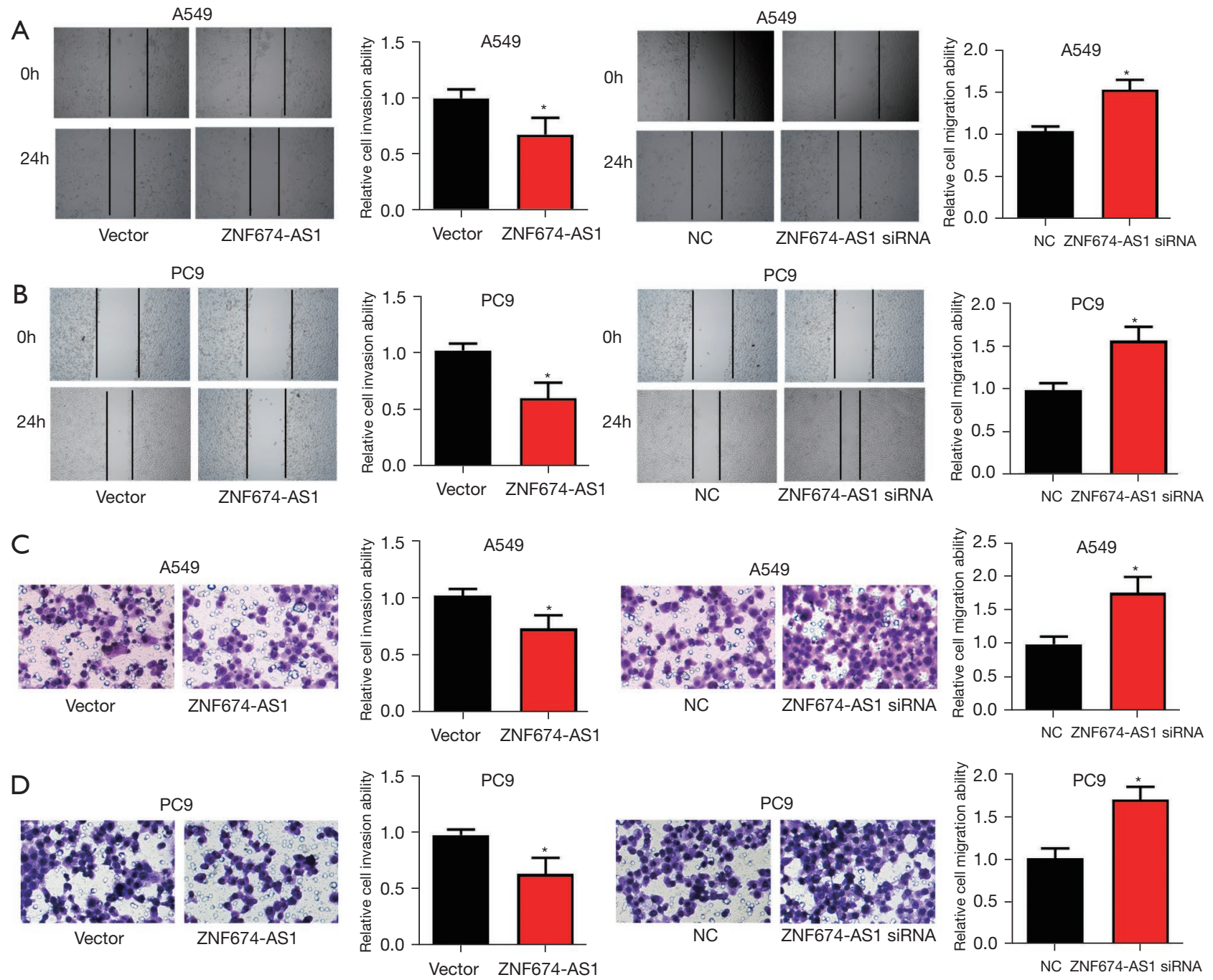

Figure 2 ZNF674-AS1 inhibits the migration and invasion of NSCLC cells. (A,B) Scratch assay was used to study the effects of overexpression and silencing of ZNF674-AS1 on NSCLC cell migration. (C,D) Transwell assay was used to study the effects of overexpression and silencing of ZNF674-AS1 on NSCLC cell invasion. Cells were stained with $0.5 \%$ crystal violet solution; magnification, 100x. *, $\mathrm{P}<0.05$. NSCLC, non-small cell lung cancer.

\section{miR-23a can directly inbibit the expression of E-cadherin}

Next, we looked for downstream targets of miR-23a. Through searching the Starbase database, we predicted E-cadherin to be one of the direct downstream targets of miR-23a (Figure 5A). The predicted binding sequence between miR-23a and the E-cadherin 3'UTR was confirmed by dual-luciferase reporter gene experiment (Figure 5B). Further, we demonstrated that overexpression of miR-23a significantly inhibited E-cadherin expression in NSCLC cells, while its knockdown had the opposite effect (Figure 5C,5D). These results indicated that miR-23a could directly inhibit the expression of E-cadherin in NSCLC cells.

\section{Discussion}

The malignant process of NSCLC is extremely complex, and its molecular mechanism is unclear. In recent years, non-coding RNAs have increasingly drawn attention for their role in tumor development. The current study found that the expression of lncRNA ZNF674-AS1 was significantly reduced in NSCLC tissues and cell lines, and in vitro experiments showed that ZNF674-AS1 could inhibit 

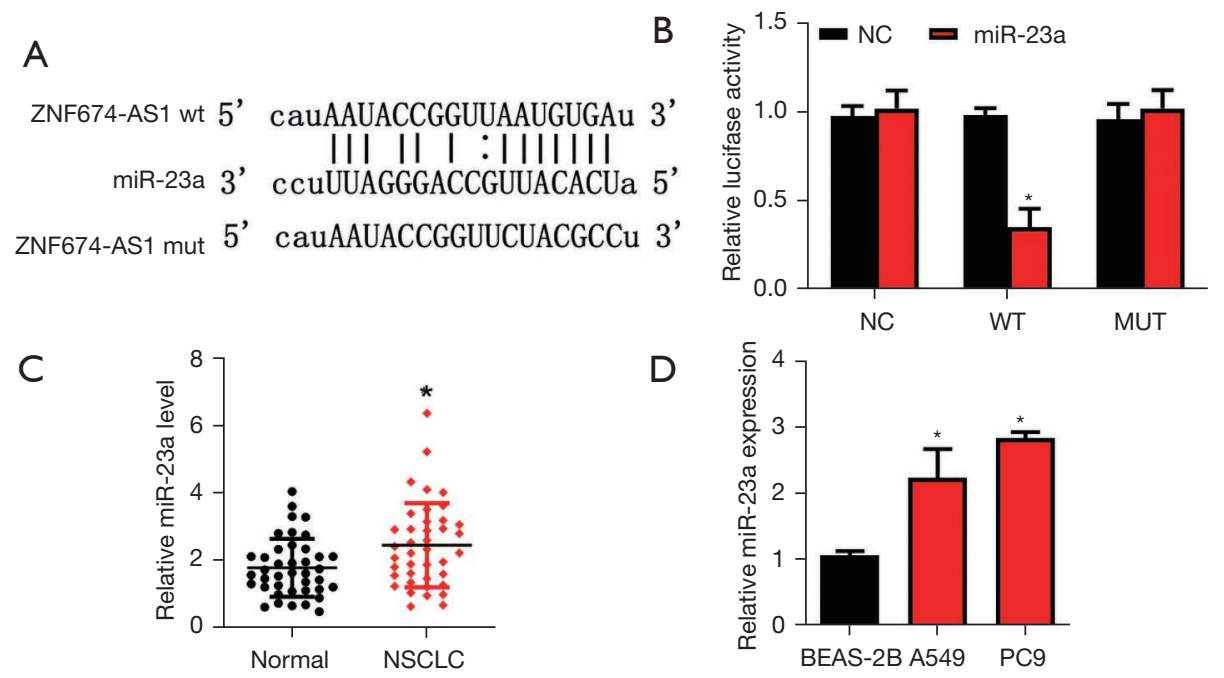

Figure $3 \mathrm{miR}-23 \mathrm{a}$ is the downstream target of ZNF674-AS1. (A) The binding sites of miR-23a and ZNF674-AS1 were predicted with Starbase. (B) The predicted binding sites of miR-23a and ZNF674-AS1 were verified by dual-luciferase reporter gene assay. (C) The expression of miR-23a was detected by qRT-PCR. (D) The expression of miR-23a in NSCLC cell lines A549 and PC9 and standard lung epithelial cell line BEAS-2B was detected by qRT-PCR. * $\mathrm{P}<0.05$. qRT-PCR, quantitative real-time polymerase chain reaction; NSCLC, non-small cell lung cancer.

the migration and invasion of NSCLC cells.

Previous studies have reported that ZNF674-AS1 is downregulated in liver cancer tissues and cell lines, with its expression level being significantly related to tumor metastasis, clinical stage, histopathological classification, and prognosis (13). In Zhang et al.'s study, Kaplan-Meier survival curve analysis showed that a low expression of ZNF674-AS1 in liver cancer was associated with a poor prognosis (13). Furthermore, a recent study showed a decreased expression of ZNF674-AS1 in NSCLC (11). The downregulation of ZNF674-AS1 was found to be significantly related to the TNM stage and overall survival rate of patients with NSCLC (11). ZNF674-AS1 can inhibit NSCLC cell proliferation, colony formation, and tumorigenesis by inhibiting the cell cycle block of G0/ G1 cells (11). Therefore, the results of our study not only partially confirm the results of previous works but also supplement their findings.

Previous studies have shown that ZNF674-AS1 can play a biological role by regulating of miR-423-3p . In this study, miR-23a was identified as a target of ZNF674-AS1. The expression of miR-23a was significantly higher in NSCLC than in the adjacent lung tissues. Qu et al. found that miR23a expression was related to smoking status, tumor size, lymph node metastasis, TNM stage, tumor differentiation, and overall survival in patients with NSCLC, and was an independent prognostic factor (12). Our study also showed that miR-23a expression was increased in both NSCLC tissues and cell lines, which was consistent with previous research results. In terms of its biological activity, miR-23a has been reported to promote the migration and invasion of NSCLC cells and reduce the sensitivity of lung cancer stem cells to erlotinib through the PTEN/PI3K/Akt pathway $(14,15)$. The present study showed that miR-23a could antagonize the effects of ZNF674-AS1 and promote NSCLC cell migration and invasion, which is consistent with previous research findings.

Previous studies showed that lncRNA ZNF674-AS1 could promote $\mathrm{p} 21$ expression by antagonizing miR-423-3p $(11,16,17)$. As an important cell cycle regulator, p21 induces the cell cycle arrest of G0/G1 NSCLC cells to inhibit tumor growth $(11,16,17)$. In this study, we confirmed that miR23a targets and regulates E-cadherin directly . E-cadherin, the key inhibitory factor in epithelial-mesenchymal transformation, can negatively regulate the migration and invasion of many tumor-related biological processes. Insufficient expression of E-cadherin is a predictor of metastasis and a poor prognosis of multiple cancer (18-20). Therefore, we believe that the ZNF674-AS1/miR-23a signaling axis regulates the migration and invasion of 

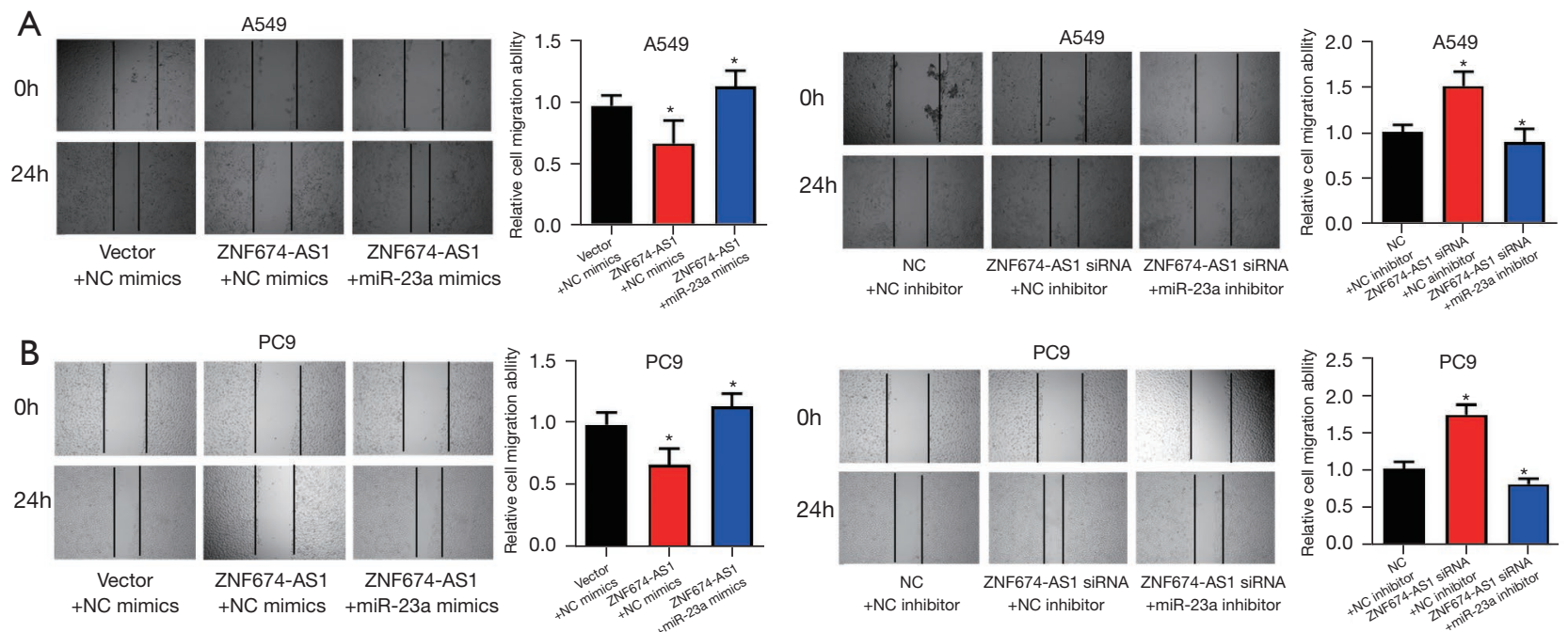

PC9
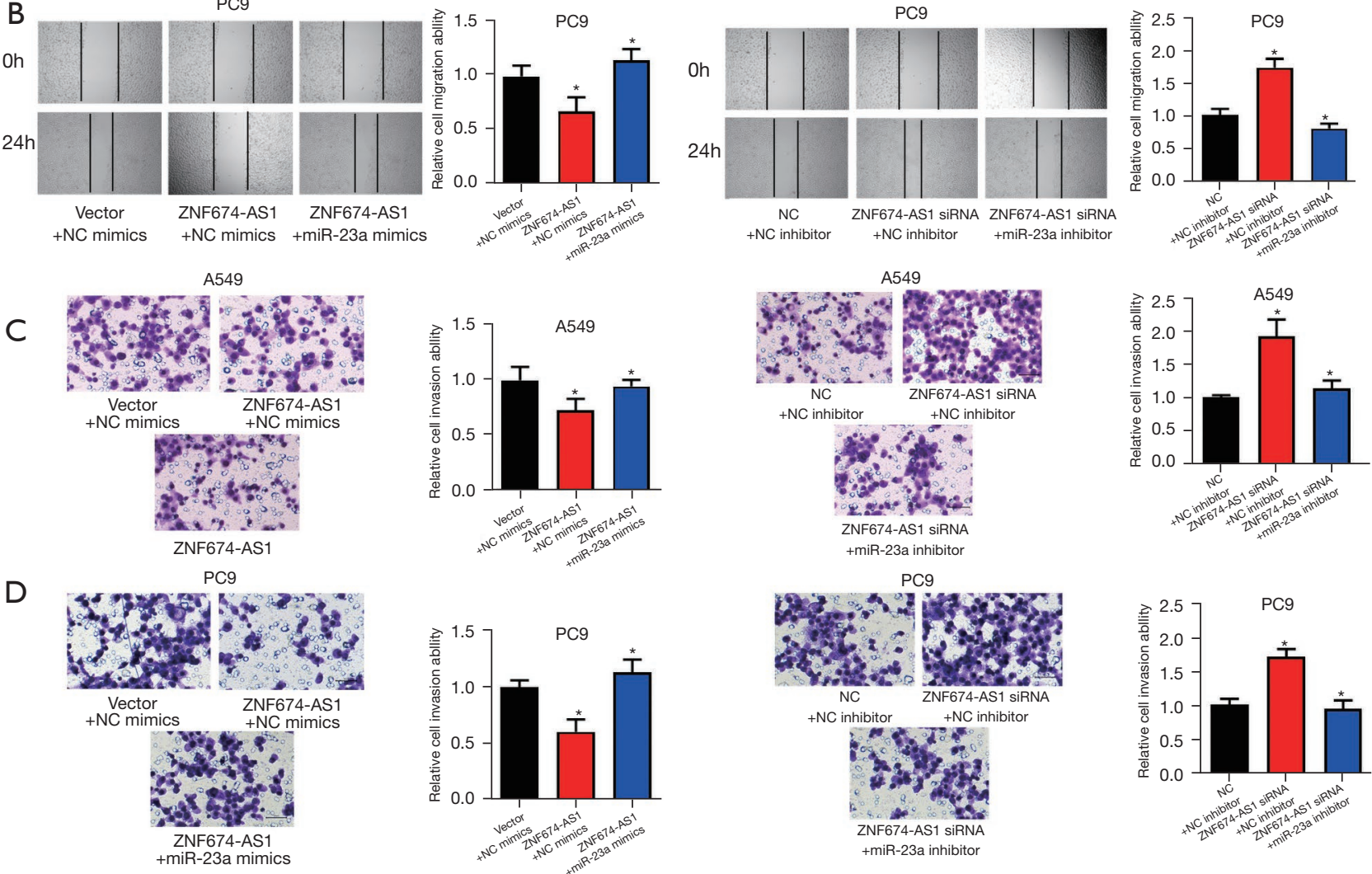

Figure 4 miR-23a antagonizes the role of ZNF674-AS1 in NSCLC. (A,B) A scratch test was conducted to study the effects of ZNF674-AS1 and miR-23a on NSCLC cell migration. (C,D) A Transwell experiment was conducted to study the impact of ZNF674-AS1 and miR-23a on NSCLC cell invasion. Cells were stained with $0.5 \%$ crystal violet solution; magnification, $100 \times$. *, P<0.05. NSCLC, non-small cell lung cancer. 

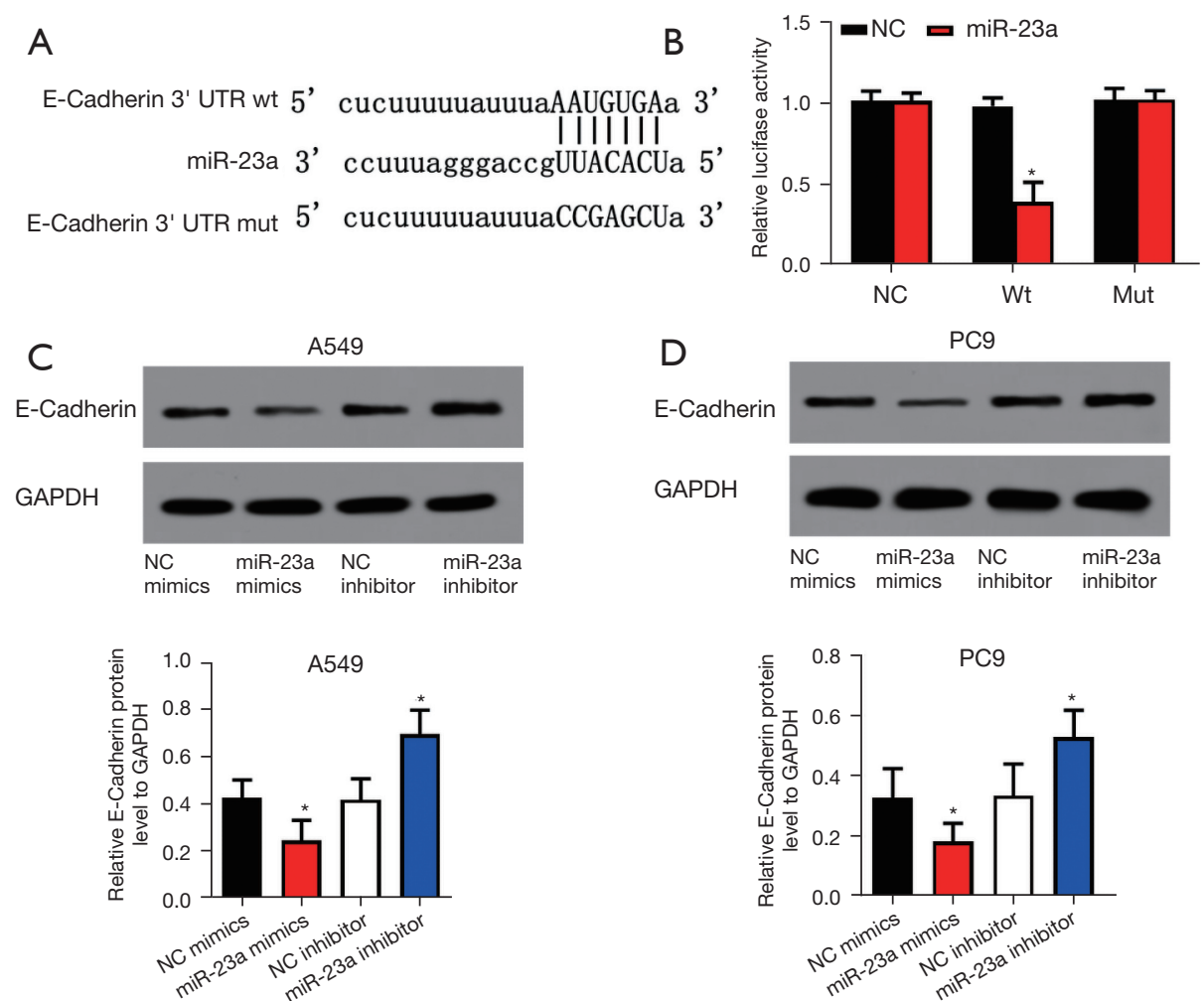

Figure 5 miR-23a can directly inhibit E-cadherin expression. (A) The binding sites of miR-23a and the E-cadherin 3'UTR were predicted with Starbase. (B) A dual-luciferase reporter gene assay was used to verify the predicted binding site of miR-23a and E-cadherin 3'UTR. (C,D) Western blot was used to detect the effects of miR-23a overexpression and silencing on E-cadherin expression in NSCLC cells. * $\mathrm{P}<0.05$. 3'UTR, three prime untranslated region; NSCLC, non-small cell lung cancer.

NSCLC cells by regulating E-cadherin expression.

In conclusion, this study has shown that ZNF674-AS1 and miR-23a are abnormally expressed in NSCLC tissues and cells. ZNF674-AS1 inhibits NSCLC cell migration and invasion by regulating miR-23a/E-cadherin. These results suggest that ZNF674-AS1 and miR-23a may be potential therapeutic targets for NSCLC.

\section{Acknowledgments}

Funding: This study was supported by Hainan Innovation Team Project (grant No. CXTD448), Hainan Provincial Key Research and Development Project (grant No. ZDYF2020223), the National Natural Science Foundation of China (grant No. 81860001 and 82011530049), the Scientific Research Project of Health and Family Planning Industry in Hainan Province (19A200036), the National Natural Science Foundation of Hainan Province (Grant No.818QN318), and Hainan Provincial Natural Science
Fundation of China (820CXTD448).

\section{Footnote}

Reporting Checklist: The authors have completed the MDAR reporting checklist. Available at https://dx.doi. org/10.21037/tcr-21-1499

Data Sharing Statement: Available at https://dx.doi. org/10.21037/tcr-21-1499

Conflicts of Interest: All authors have completed the ICMJE uniform disclosure form (available at https://dx.doi. org/10.21037/tcr-21-1499). The authors have no conflicts of interest to declare.

Ethical Statement: The authors are accountable for all aspects of the work in ensuring that questions related to the accuracy or integrity of any part of the work are 
appropriately investigated and resolved. All procedures performed in this study involving human participants were in accordance with the Declaration of Helsinki (as revised in 2013). The study was approved by the Ethics Committee of the First Affiliated Hospital of Hainan Medical University and informed consent was taken from all the patients (No.: 2019-57).

Open Access Statement: This is an Open Access article distributed in accordance with the Creative Commons Attribution-NonCommercial-NoDerivs 4.0 International License (CC BY-NC-ND 4.0), which permits the noncommercial replication and distribution of the article with the strict proviso that no changes or edits are made and the original work is properly cited (including links to both the formal publication through the relevant DOI and the license). See: https://creativecommons.org/licenses/by-nc-nd/4.0/.

\section{References}

1. Carlisle JW, Steuer CE, Owonikoko TK, et al. An update on the immune landscape in lung and head and neck cancers. CA Cancer J Clin 2020;70:505-17.

2. Iams WT, Porter J, Horn L. Immunotherapeutic approaches for small-cell lung cancer. Nat Rev Clin Oncol 2020;17:300-12.

3. Arbour KC, Riely GJ. Systemic Therapy for Locally Advanced and Metastatic Non-Small Cell Lung Cancer: A Review. JAMA 2019;322:764-74.

4. Spitaleri G, Passaro A, de Marinis F. Ensartinib (X-396) a novel drug for anaplastic lymphoma kinase-positive nonsmall cell lung cancer patients: we need smart trials to avoid wasting good bullets. Chin Clin Oncol 2019;8:S1.

5. Herbst RS, Morgensztern D, Boshoff C. The biology and management of non-small cell lung cancer. Nature 2018;553:446-54.

6. Wang M, Sun X, Wang H, et al. Long non-coding RNAs in non-small cell lung cancer: functions and distinctions from other malignancies. Transl Cancer Res 2019;8:2636-53.

7. Statello L, Guo CJ, Chen LL, et al. Gene regulation by long non-coding RNAs and its biological functions. Nat Rev Mol Cell Biol 2021;22:96-118.

8. Goodall GJ, Wickramasinghe VO. RNA in cancer. Nat Rev Cancer 2021;21:22-36.

9. Yao G, Chen K, Qin Y, et al. Long Non-coding RNA JHDM1D-AS1 Interacts with DHX15 Protein to Enhance Non-Small-Cell Lung Cancer Growth and Metastasis.
Mol Ther Nucleic Acids 2019;18:831-40.

10. Wang H, Lu B, Ren S, et al. Long Noncoding RNA LINC01116 Contributes to Gefitinib Resistance in Nonsmall Cell Lung Cancer through Regulating IFI44. Mol Ther Nucleic Acids 2020;19:218-27.

11. Liu Y, Huang R, Xie D, et al. ZNF674-AS1 antagonizes miR-423-3p to induce G0/G1 cell cycle arrest in nonsmall cell lung cancer cells. Cell Mol Biol Lett 2021;26:6.

12. Qu WQ, Liu L, Yu Z. Clinical value of microRNA-23a upregulation in non-small cell lung cancer. Int J Clin Exp Med 2015;8:13598-603.

13. Zhang L, He T, Yan Y, et al. Expression and Clinical Significance of the Novel Long Noncoding RNA ZNF674-AS1 in Human Hepatocellular Carcinoma. Biomed Res Int 2016;2016:3608914.

14. Cao M, Li Y, Lu H, et al. MiR-23a-mediated migration/ invasion is rescued by its target, IRS-1, in nonsmall cell lung cancer cells. J Cancer Res Clin Oncol 2014;140:1661-70.

15. Han Z, Zhou X, Li S, et al. Inhibition of miR-23a increases the sensitivity of lung cancer stem cells to erlotinib through PTEN/PI3K/Akt pathway. Oncol Rep 2017;38:3064-70.

16. Xie D, Lan L, Huang K, et al. Association of p53/p21 expression and cigarette smoking with tumor progression and poor prognosis in non-small cell lung cancer patients. Oncol Rep 2014;32:2517-26.

17. Gupta S, Silveira DA, Mombach JCM. ATM/miR-34a$5 \mathrm{p}$ axis regulates a $\mathrm{p} 21$-dependent senescence-apoptosis switch in non-small cell lung cancer: a Boolean model of G1/S checkpoint regulation. FEBS Lett 2020;594:227-39.

18. Corso G, Figueiredo J, De Angelis SP, et al. E-cadherin deregulation in breast cancer. J Cell Mol Med 2020;24:5930-6.

19. Sommariva M, Gagliano N. E-Cadherin in Pancreatic Ductal Adenocarcinoma: A Multifaceted Actor during EMT. Cells 2020;9:1040.

20. Biswas KH. Molecular Mobility-Mediated Regulation of E-Cadherin Adhesion. Trends Biochem Sci 2020;45:163-73.

(English Language Editor: J. Reynolds)

Cite this article as: Wang J, Liu S, Pan T, Wang M, Li L, Weng X, Jia P, Li Q, Zhou X. Long non-coding RNA ZNF674-AS1 regulates $\mathrm{miR}-23 \mathrm{a} / \mathrm{E}$-cadherin axis to suppress the migration and invasion of non-small cell lung cancer cells. Transl Cancer Res 2021;10(9):4116-4124. doi: 10.21037/tcr-21-1499 